\title{
El método de estudio de casos en la enseñanza de las ciencias naturales
}

Angie Viviana Arango Martínez ${ }_{\text {Universidad Pedagógica y Tecnológica de Colombia, Colombia }}^{1{ }^{(0)}}$

\section{Resumen}

La enseñanza de las ciencias naturales debe integrar el contexto para fortalecer las habilidades de los estudiantes. De acuerdo con esto, las diversas estrategias y metodologías que los docentes utilizan para orientar las clases y para reflexionar sobre su práctica los llevan a mejorar su labor y a alcanzar un aprendizaje significativo en los educandos. En el presente artículo, se da a conocer una investigación de carácter cualitativo, con el objetivo de diseñar e implementar el método de estudio de casos en la enseñanza de las ciencias naturales, para realizar una aproximación hacia la integración de áreas en la escuela rural multigrado a partir del estudio de las aves del municipio de Toca, Boyacá -Colombia-. Se involucró a 14 participantes de básica primaria. Los resultados muestran que el estudio de casos promueve la apropiación de aptitudes hacia el cuidado de las aves, la enseñanza holística de las ciencias y la formulación de preguntas que motivan el análisis y la reflexión. Así, el estudio de casos proporciona herramientas de apoyo al docente de escuela rural multigrado en su labor de enseñanza.

Palabras clave: enseñanza, estudio de caso, ciencias naturales, educación rural 


\title{
The case study method in the teaching of the natural sciences
}

\begin{abstract}
Natural science teaching must involve the context in order to strengthen students' skills. Thus, the various strategies and methodologies that teachers use to guide classes and to reflect on their practice lead them to improve their work and their students' meaningful learning. This article presents a qualitative research, with the objective of designing and implementing the case study method in the teaching of natural sciences, aiming to provide an approach towards the integration of subjects in the multigrade rural school with the study of birds in the municipality of Toca, Boyacá-Colombia. Fourteen primary school participants were involved. The results show that the case study promotes the appropriation of skills towards the care of birds, the holistic teaching of science, and the formulation of questions that stimulate analysis and reflection. Hence, the case study provides tools to support multigrade rural schoolteachers in their teaching work.
\end{abstract}

Keywords: teaching, case study, natural sciences, rural education

\section{O método de estudo de casos no ensino das ciências naturais}

\section{Resumo}

O ensino das ciências naturais deve integrar o contexto para reforçar as competências dos estudantes. De acordo com isto, as várias estratégias e metodologias que os professores utilizam para orientar as aulas e para refletir sobre a sua prática os levam a melhorar o seu trabalho e a alcançar uma aprendizagem significativa nos estudantes. Este artigo apresenta uma pesquisa qualitativa, com o objetivo de conceber e implementar o método de estudo de casos no ensino das ciências naturais, para fazer uma abordagem à integração das áreas na escola rural multigradual a partir do estudo das aves no município de Toca, Boyacá - Colômbia. 14 participantes do ensino básico primário foram envolvidos. Os resultados mostram que o estudo de casos promove a apropriação de aptidões para o cuidado das aves, o ensino holístico das ciências e a formulação de questões que motivam a análise e a reflexão. Assim, o estudo de casos fornece ferramentas para apoiar o professor de escola rural multigradual em seu trabalho de ensino.

Palavras-chave: ensino, estudo de caso, ciências naturais, educação rural

Según la investigación científica (Abós, 2015; Arriagada \& Calzadilla, 2018; Bustos, 2014; Faure, 2017; Rivera \& Zabala, 2019), la escuela rural en Colombia ha sido tema de diversos estudios enfocados a definir y analizar sus características (Arias, 2017; Herrera \& Buitrago, 2015; Ministerio de Educación Nacional [MEN], 2010; Soto \& Molina, 2018). Algunos autores han enfatizado en los estudiantes y en sus concepciones de aprendizaje 
(Abós et al., 2017; Lacruz et al., 2017). Otros investigaron sobre las implicaciones de la labor docente (Ramírez et al., 2019). Además, se destacan los retos que la educación rural en Iberoamérica tiene en torno a adaptabilidad, accesibilidad, aceptabilidad y disponibilidad, con el fin de promover el derecho a la educación de calidad (Rivera \& Zabala, 2019). En este sentido, Lara y Pulido (2020) mencionan que la escuela y el contexto rural brindan diversas oportunidades para orientar procesos de enseñanza, por lo que sus características y particularidades no siempre deben verse como dificultades (Arriagada \& Calzadilla, 2018).

En Colombia, según el MEN (2002), la educación rural, en su mayoría, se orienta por medio de aulas multigrado. Es decir, son escuelas en las cuales un docente enseña todas las áreas del currículo a uno o más grados al mismo tiempo (Faure, 2017). Por lo tanto, y según Bustos (2014) y Faure (2017), los docentes deben diseñar e implementar estrategias didácticas de enseñanza como: la utilización de proyectos o talleres que posibiliten abordar diferentes áreas simultáneas; el involucrar el contexto o medio escolar; integrar los saberes empíricos de la comunidad para generar aprendizajes en los estudiantes; acudir a los monitoreos entre alumnos; organizar el agrupamiento multigrado; y utilizar secuencias didácticas conjuntamente para diferentes grados. Lo anterior permite responder a la heterogeneidad, sin que prevalezcan las visiones discriminativas.

En este sentido, el MEN, en Colombia, desde 1976, creó el Modelo de Escuela Nueva (MEN, 2002, 2010; Soto \& Molina, 2018), el cual ha sido adoptado por diferentes países y se ha convertido didáctica, metodológica y pedagógicamente en un apoyo para la educación en escuelas rurales multigrado (Colbert, 1999; MEN, 2010; Rivera \& Zabala, 2019). Teniendo en cuenta las fortalezas del modelo de Escuela Nueva para favorecer una educación de calidad en zonas rurales en modalidad multigrado, situaciones como el afán por cumplir el plan de estudios y la dificultad de manejar diferentes edades y grados llevan a que los estudiantes terminen completando guías sin comprender, en muchas ocasiones, realmente lo que hacen, ya que prestan poca atención al contexto y al desarrollo de habilidades. Lo apropiado sería acompañar el modelo con estrategias didácticas que no lleven solo al aprendizaje de contenidos y competencias básicas, sino también a la formación integral de la persona, que incluye destrezas, valores y actitudes (Abós et al., 2017).

\section{El estudio de casos como método de enseñanza}

El estudio de casos como método de enseñanza es diferente al estudio de caso como método de investigación (Jiménez \& Comet, 2016), pues los resultados del segundo pueden utilizarse como un caso de estudio en las sesiones de clase. Además, el primero requiere del planteamiento de objetivos de aprendizaje al inicio de su aplicación para poder evaluar su eficacia (Díaz et al., 2011). El método del caso surge en la universidad de Harvard hacia 1914 y es llamado "estudio de casos" (Wassermann, 1994) o "aprendizaje basado en el análisis de casos" (Díaz \& Hernández, 1999). Es un método que goza de una naturaleza interdisciplinar al favorecer el aprendizaje en diversas áreas y niveles educativos, así como la adquisición de conocimientos y el desarrollo de habilidades y actitudes (Wassermann, 1994). En este sentido, un caso es una historia real que plantea problemas para que los estudiantes comprendan situaciones complejas (Barros, 2019). Como lo menciona Wassermann (2006), "un caso es el vehículo por medio del cual se lleva al aula un trozo de realidad a fin de que los alumnos y el profesor lo examinen minuciosamente" (p. 20). 
Así mismo, incluir el método de estudio de casos [MEC] en las sesiones de clase promueve el aprendizaje de tópicos reflexivamente, ya que los estudiantes pueden comprender e interpretar el caso, analizarlo contextualmente e intervenir con preguntas o propuestas sobre este de una manera adecuada (Gamboa, 2017; Gutiérrez et al., 2019). Según Niño (2012) y Niño y Pedraza (2019), el estudiar un caso favorece la construcción del conocimiento basado en la indagación de situaciones contextuales. Por ejemplo, se pueden analizar problemas que no son ajenos a la cotidianidad del estudiante.

Además de las bondades ya mencionadas, diferentes autores que han utilizado el MEC en sus clases (Argandoña et al., 2018; Gutiérrez et al., 2019; Niño, 2012; Niño \& Pedraza, 2019; Passos et al., 2007; Somma, 2012; Wassermann, 2006) exponen situaciones que caracterizan la enseñanza como:

- El narrar historias actuales, cortas, reales e interesantes que favorecen el desarrollo del pensamiento crítico, la creatividad, el trabajo en equipo, la toma de decisiones, la innovación, el respeto, la colaboración, la responsabilidad y la tolerancia.

- El manejo y formulación de preguntas críticas son la clave para lograr la reflexión y análisis de las situaciones problema.

- Su metodología involucra actividades de seguimiento diseñadas por el docente.

- Entre sus propósitos, está que los estudiantes aprendan para la vida.

- Se fortalecen habilidades de síntesis, escritura, análisis, evaluación de la información y comunicación.

- El papel del docente es el de mediar las discusiones.

- El estudiar casos promueve la comprensión de tópicos o áreas estudiadas por parte de los estudiantes.

Las investigaciones encontradas en relación con el estudio de casos como método de enseñanza de las ciencias naturales en Colombia son pocas. Sin embargo, luego de una revisión bibliográfica en revistas indexadas de los últimos diez años -2010-2020 -, se identificaron ocho estudios que involucran el MEC para diseñar estrategias y unidades didácticas. Están clasificados en artículos (Niño, 2012; Niño \& Pedraza, 2019; Revel, 2013), memorias de eventos científicos (Barros, 2019; Gamboa, 2017; Gil \& Barreto, 2019) y tesis de maestría (Barros et al., 2017; Chin, 2013). La mayoría de estas investigaciones se realizaron con población de básica secundaria y de educación superior. Concluyen que el método proporciona diversas posibilidades (tabla 1) en la enseñanza de las ciencias.

Tabla 1

Posibilidades del MEC en las investigaciones realizadas en Colombia en el periodo 2010-2020 sobre la enseñanza de las ciencias naturales

Posibilidades de su implementación en la enseñanza de las ciencias naturales

Diseñar estrategias didácticas para enseñar conceptos de las ciencias naturales: sexualidad, fisiología humana, prevención y salud, biología, fotosíntesis y respiración de plantas, sistema nervioso y nicho ecológico.

Promover el trabajo colaborativo y participativo en el aula, al igual que actitudes positivas para los procesos de enseñanza.
Número de estudios

8 
Desarrollo de procesos de pensamiento crítico: síntesis, análisis y reflexión para proponer y comprender situaciones.

6

Desarrollo de competencias científicas como la indagación y argumentación. Alfabetización científica.

Uso interdisciplinario para comprender situaciones científicas contextualizadas.

Fomentar la educación ambiental

Fomentar procesos de escritura y lectura.
5

4

2

1

Nota: Para una comprensión adecuada de la información que contiene esta tabla, es importante aclarar que la sumatoria de los números no responde al total de documentos analizados — - - dado que a cada estudio se le asignó más de una posibilidad.

Ahora bien, el análisis de los ocho documentos permitió resaltar las posibilidades más representativas que el MEC - en dicho lapso - tiene en el aprendizaje y comprensión de conceptos de las ciencias naturales, así como en el trabajo cooperativo, en el desarrollo de competencias científicas y en el pensamiento crítico.

Entonces, el interés de realizar esta investigación viene dado por la necesidad de articular la enseñanza en aulas multigrado con el desarrollo de habilidades, aptitudes y procedimientos que incluyan el estudio del contexto rural, para solventar las dificultades didácticas y pedagógicas con las que el docente se encuentra, como el orientar todas las áreas en los diferentes niveles educativos al mismo tiempo. Para ello, se implementa el MEC, pues, según la revisión bibliográfica, este permite promover el aprendizaje de conceptos contextualizados y fomentar el desarrollo de habilidades como: síntesis, discusión, análisis, formulación de preguntas y reflexión. Además, se convierte en una herramienta de enseñanza para el docente de aula multigrado, al tener una naturaleza de integralidad de áreas.

La investigación tuvo como finalidad implementar el MEC en la enseñanza de las ciencias naturales en el contexto rural de básica primaria para: fomentar espacios de discusión, orientar procesos que promuevan una enseñanza integral de diversas áreas en el aula multigrado, proponer actividades que fortalezcan habilidades de pensamiento crítico y aprender sobre la avifauna en torno a su morfología, comportamiento e importancia.

\section{Metodología}

Esta investigación se desarrolló a lo largo del 2019, con una metodología cualitativa. Se partió de la observación y la descripción de los datos para su adecuado análisis (Hernández et al., 2014). El MEC, según Wassermann (2006), es escogido para diseñar e implementar la estrategia didáctica. En este proyecto participaron catorce estudiantes de grado segundo a quinto de básica primaria entre los 6 y 12 años, pertenecientes a la Institución Educativa Rural Técnica Rafael Uribe, sede Leonera de Toca, Boyacá, los cuales firmaron consentimientos y asentimientos requeridos para el manejo de datos.

Las fases determinadas por Wassermann (2006) sobre el MEC que se adoptaron en la estrategia didáctica fueron: diseño, lectura, discusión en pequeños grupos, discusión general y actividades de seguimiento (figura 1). La estrategia estuvo conformada por tres talleres que involucraron el estudio y el análisis contextual de tres casos - sometidos a validación de expertos en el tema-. 


\section{Diseño de caso}

Los casos involucraron como protagonistas especies de aves del entorno con el fin de promover el interés de los participantes. Además, el estudio de este grupo animal permite la enseñanza de temas de las ciencias naturales, pero, sobre todo, el desarrollo de habilidades científicas. Los casos que se diseñaron se describen a continuación:

- Caso 1. Aprendiendo con mi padre: involucra a la especie Zonotrichia capensis o copetón. Esta es un ave común de la vereda Leonera y del altiplano cundiboyacense. El caso cuenta la historia de un padre que dialoga con su hijo sobre el comportamiento de la especie, los nombres comunes que recibe en diferentes países y su alimentación.

- Caso 2. Alondrita, alondrita: cuenta la historia del ave Eremoplila alpestris, la cual es endémica para el territorio colombiano y está en peligro de extinción. Aquí la alondra misma expone las causas que la tienen en riesgo de desaparecer, como otras cuestiones de morfología, hábitat y comportamiento.

- Caso 3. Largo vuelo para vivir: el águila pescadora - Pandion heliaetus - es una especie migratoria que recorre varios kilómetros desde Norteamérica hasta Suramérica para sobrevivir a los cambios de clima, en busca de alimento. La historia describe situaciones cotidianas de la vereda - Leonera y Represa la Copa de Toca, Boyacá, Colombia - y del contexto de los estudiantes.

\section{Figura 1}

Fases del MEC desarrolladas en la estrategia

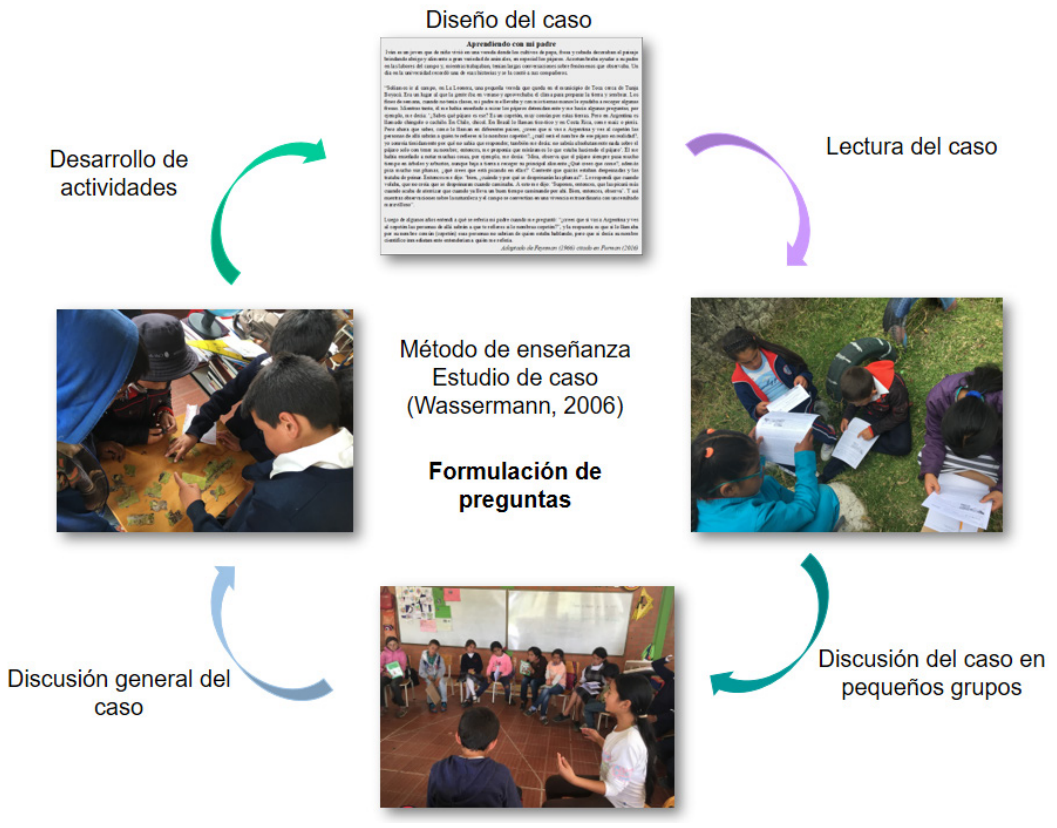

Adaptada de El estudio de casos como método de enseñanza de S. Wassermann, 2006.

\section{Lectura y discusión del caso en pequeños grupos}

En esta fase del método, los estudiantes, reunidos en pequeños grupos, realizan una primera lectura del caso. Formulan preguntas, buscan palabras desconocidas y relacionan la historia con su contexto rural. 


\section{Discusión general del caso}

Todos los estudiantes junto con el docente analizan a profundidad el caso. Primero, los estudiantes reconstruyen y sintetizan la historia. Luego, revelan las preguntas y dudas suscitadas durante su lectura. En esta fase, cobra gran importancia la capacidad del docente para guiar las intervenciones de los participantes y orientar la discusión, donde docente y estudiantes formulan preguntas con el fin de analizar las diferentes situaciones que el caso presenta.

\section{Actividades de seguimiento del caso}

Se plantean diversas actividades que llevan a profundizar en los temas identificados en la discusión, las cuales se diseñaron desde diferentes áreas del saber para aprovechar la naturaleza interdisciplinar del método.

Se realizaron grabaciones de audio sobre las discusiones de los casos para recopilar información. Además, los escritos, diarios de campo y talleres desarrollados por los estudiantes también fueron recopilados, con el fin de analizarlos a partir de códigos y categorías inductivas (Strauss \& Corbin, 2002). A su vez, se utilizó la triangulación de datos - grabaciones, documentos oficiales, talleres y diarios de campo- para darles mayor validez. Los resultados se enfocan en mostrar y analizar cómo la formulación de preguntas relaciona diversas áreas y promueve el fortalecimiento de habilidades. El diálogo y la discusión de los casos son de gran importancia para desarrollar el MEC en la enseñanza de las ciencias naturales en la escuela rural multigrado.

\section{Resultados y discusión}

El MEC, en sus diversas fases, brinda variedad de posibilidades — didácticas, pedagógicas y actitudinales- para la enseñanza en la escuela rural multigrado. Al analizar un caso en profundidad, es posible vincular diferentes saberes, lo cual le permite al docente del aula multigrado abarcar temáticas de las áreas fundamentales de la educación básica primaria. Más que enseñar temáticas, se fomenta en el estudiante el fortalecimiento de habilidades científicas. Además, investigaciones como las de Piñones y Zuleta (2014), Sanabria y Sosa (2017) y Rodríguez (2017) dan cuenta de las oportunidades educativas de estudiar las aves del contexto. Entre ellas está el desarrollo de habilidades científicas - como las que se muestran en la tabla 2- y actitudes de aprecio, curiosidad, asombro y protección hacia las aves y su entorno.

Tabla 2

Habilidades científicas fortalecidas en los estudiantes a partir del estudio de las aves

\begin{tabular}{cl}
\hline Habilidad científica & \multicolumn{1}{c}{ Ejemplo de aplicación de la habilidad } \\
\hline \multirow{2}{*}{ Observar } & $\begin{array}{l}\text { Al visualizar aves de la vereda, como el copetón, el siote, la garza, el gavilán, } \\
\text { la paloma, entre otras. }\end{array}$ \\
\hline \multirow{2}{*}{ Proponer } & $\begin{array}{l}\text { Al idear acciones para disminuir las amenazas de las aves en la vereda. Al } \\
\text { componer coplas sobre las aves estudiadas. }\end{array}$ \\
\hline \multirow{2}{*}{ Analizar } & $\begin{array}{l}\text { Al estudiar el comportamiento migratorio del águila pescadora, o al analizar } \\
\text { las diversas cuestiones planteadas en la fase de discusión de cada caso de } \\
\text { estudio. }\end{array}$ \\
\hline
\end{tabular}




\begin{tabular}{cl}
\hline Clasificar & $\begin{array}{l}\text { Al diferenciar los tipos de picos de las aves y la alimentación en la que se } \\
\text { agrupan. }\end{array}$ \\
\hline Formular preguntas & Al formular preguntas como las que se muestran en la tabla 3. \\
\hline Registrar & $\begin{array}{l}\text { Al plasmar observaciones en los diarios de campo sobre las aves, también al } \\
\text { registrar datos necesarios para resolver dudas. }\end{array}$ \\
\hline Experimentar & $\begin{array}{l}\text { Al verificar temas sobre las aves vistos en clase, con sus propias observaciones } \\
\text { realizadas en campo. }\end{array}$ \\
\hline Construir & $\begin{array}{l}\text { Llegar a definiciones de temas relacionados con las aves como: dimorfismo } \\
\text { sexual, migración, endémico, silvestre. }\end{array}$ \\
\hline Evaluar & $\begin{array}{l}\text { Al analizar los datos obtenidos en campo y en equipo para resolver la } \\
\text { cuestión: “ ¿cuál es el ave más común de la vereda?” }\end{array}$ \\
\hline Comunicar & Al expresar ante la clase las observaciones registradas en el diario de campo. \\
\hline Describir & $\begin{array}{l}\text { Al describir la morfología del ave estudiada, con el uso de conocimientos } \\
\text { sobre sus partes y funciones. }\end{array}$ \\
\hline Explorar & $\begin{array}{l}\text { Al indagar con familiares sobre coplas, agüeros, usos, importancia y } \\
\text { prevalencia de las aves de la vereda. }\end{array}$ \\
\hline
\end{tabular}

\section{El MEC: una oportunidad para integrar áreas en la enseñanza en el aula multigrado}

El análisis de cada caso tenía como objetivo vincular diferentes saberes para tener una comprensión contextualizada de los hechos. En este sentido, tanto en la fase de discusión como en la de seguimiento, se promovió el desarrollo de actividades que llevaran a abordar cuestiones de matemáticas, lenguaje, ciencias sociales y ciencias naturales. En la discusión de los casos, entre preguntas, justificaciones, respuestas y suposiciones de los estudiantes, se develan aquellas inquietudes que vinculan diversos conocimientos. Así mismo, se reconocen los ítems para trabajar en el análisis del caso. El siguiente fragmento del estudio de Largo vuelo para vivir (Arango, 2020) es un ejemplo de la discusión dada entre docente [D] y participantes - se han utilizado seudónimos en los nombres de los estudiantes para guardar su identidad-:

—D: Bueno, pero ¿de qué más trataba la historia, de dónde venía ella, o ella siempre estaba aquí?

- Patricia: Profe, ella era migratoria.

—D: Bueno, y ¿qué entienden por migración?

- Patricia: Que pasa de un lado a otro.

—D: De un lado, ¿cómo así?

- Patricia: Que pasa en varios países.

- Santiago: Migratoria, deja un lugar y se va para otro, por ejemplo, deja un país y se va para otro país.

— D: Y ¿por qué deja el país?

- Fabián: Porque de pronto no hay alimento, o se están extinguiendo, o de pronto las matan.

— Manuel: Profe, que el águila se va para otro país porque hace frío y no pueden agarrar sus peces.

- Víctor: Se va para otros países donde hay clima, donde puede sobrevivir.

- Julián: Que los lagos se congelan y por eso las águilas se van.

- Alejandro: O porque las matan.

— D: Pero ¿qué decía la historia? Está relacionada con lo que dijo Julián, que los lagos se 
congelaban. ¿De dónde venía el águila pescadora?

— Fabián: De las zonas frías, de los nevados páramos.

— D: Presten atención niños, voy a leer el fragmento de la historia de nuevo [...] Bueno, según esto ¿de dónde venían?

— Santiago: Desde Canadá y Estados Unidos.

— D: Muy bien, ellas se venían porque allá iniciaba la temporada de invierno y los lagos se congelaban entonces era muy difícil que consiguieran alimento. Entonces ¿hacia dónde se iban?

- Manuel: Hacia Boyacá, Cundinamarca, la Patagonia.

— D: Sí, pero en la historia también decía que hacia países de Suramérica. ¿Cómo cuales países serían?

— Fabián: Brasil, Chile, Argentina, Colombia, Venezuela, Ecuador.

— D: Eso, muy bien. Entonces ellas vienen desde Norteamérica, bajan, bajan, hasta la Patagonia argentina. Ahorita vamos a ver en el mapa para ubicarnos mejor. ¿Qué más trataba la historia?

- Patricia: Profe, voy a empezar desde el inicio. Helena era una niña que le gustaba ir al embalse en las mañanas, a la Copa, a acompañar a su papá y a los demás pescadores. Entonces, ella estaba sentada alrededor. Entonces, vio que un ave más grande que un gavilán se sumergía en el agua y sacó un pez [...]

— D: Espera, Patricia. ¿Como qué gavilán conocen ustedes?

— William: Como el que tiene Alejandro en su libreta.

— D: Muy bien. ¿Cómo es que se llama su gavilán, Alejandro?

- Alejandro: Elanus leucorus.

— D: Muy bien, entonces el águila es más grande que ese gavilán. Bueno, ¿y ustedes no han visto sobre la represa (embalse la Copa) un águila?

— Varios niños: Sí, sí, sí, sí, profe.

— D: ¿Y la ven durante todo el año?

- Varios niños: No, profe. A veces.

- Manuel: Cuando no hay pescados se va para otro país.

- D: Es decir, cuando cambia el clima.

- Santiago: Y, profe, ¿cómo saben que el águila es más grande que un gavilán?

- Alejandro: Porque el gavilán es cómo de este tamaño y el águila se parece de grande como un chulo.

— Víctor: Sí, además la historia decía que el tamaño del águila era de 52 a 60 centímetros.

—D: Y ¿cuánto sería eso?

- Víctor: Eso es como el tamaño de dos reglas.

— D: Muy bien, esa relación estuvo bien. Ahora sí, continúa Patricia. (p. 76)

En este sentido, la investigación comprobó que implementar el MEC en la enseñanza de las ciencias naturales permite hacer una aproximación hacia la integración de áreas en la escuela rural, al abordar varios temas relacionados con la avifauna como: migración, comportamiento, morfología, dimorfismo sexual, nombres científicos, alimentación, funciones ecológicas, amenazas, entre otros. Sin embargo, desde una perspectiva más profunda, se emplean herramientas que brindan todas las áreas para enriquecer y darle sentido al conocimiento. Por ejemplo, al trabajar desde las ciencias naturales para 
comprender los hábitats de las aves, se abordaron conocimientos de geografía para localizar y ubicar coordenadas. Además, se emplearon elementos de matemáticas para responder a la pregunta: “¿cuál es el ave más común de mi vereda?”, al registrar y organizar datos, y así desarrollar adiciones que llevaran a la respuesta. Construir definiciones, escribir cartas, componer coplas y comunicar resultados fueron acciones que se promovieron desde el lenguaje que permitieron el fomento de habilidades lingüísticas como la expresión escrita y oral y la comprensión lectora y auditiva, a partir de la creatividad de cada uno de los participantes, en toda la implementación de la estrategia. De tal manera, los estudiantes lograron expresar en sus propias palabras la relación que tenían las formas de los picos de las aves con su tipo de alimentación y la función ecológica que cumplían (figura 2). Esto coincide con lo mencionado por Martínez (2007). El fortalecimiento de la competencia escritural evidencia una conexión entre teoría y práctica a través de la elaboración de textos de calidad que involucraron la realidad del contexto y la experiencia.

\section{Figura 2}

Formas de picos, tipos de alimentación y función ecológica de las aves en palabras de los participantes

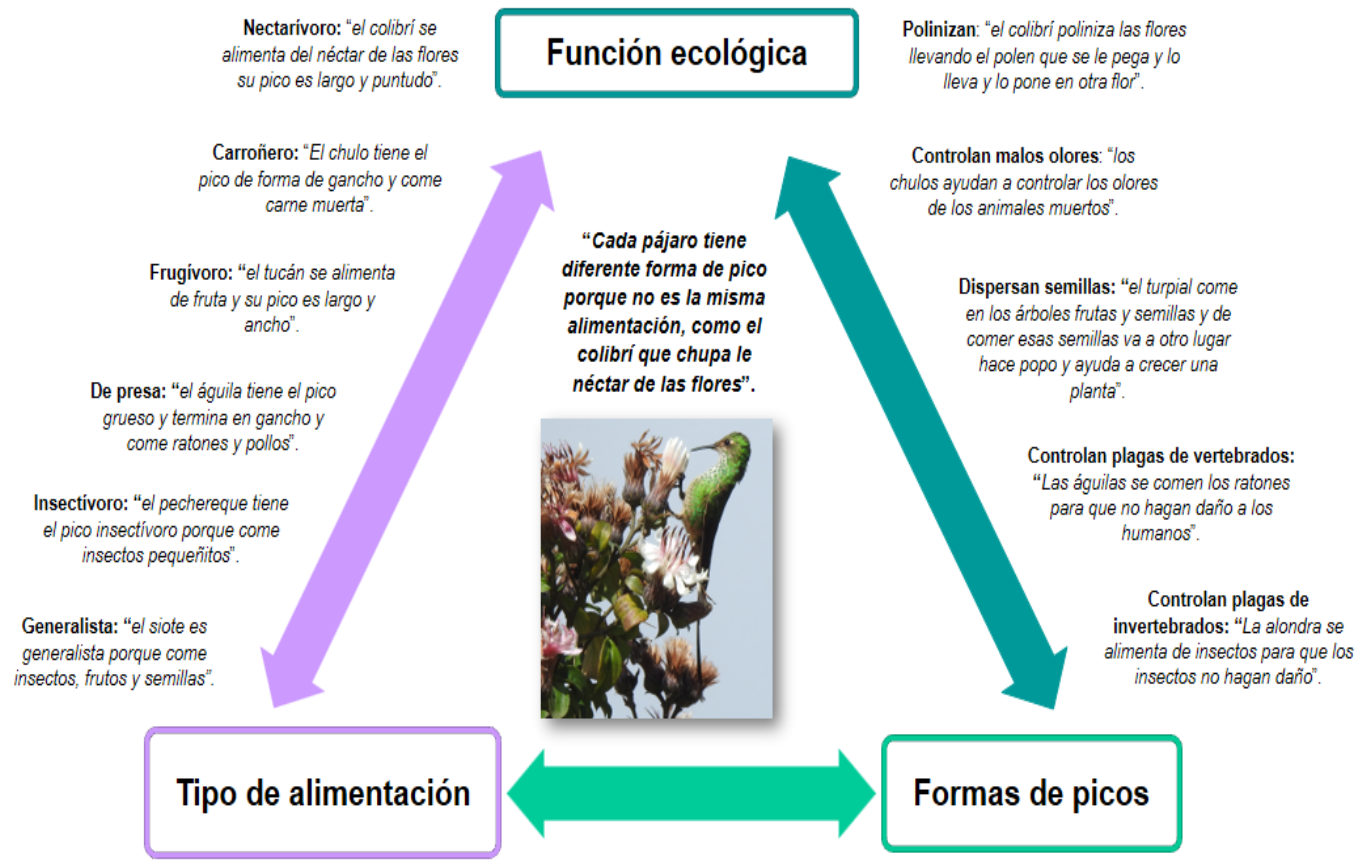

Revel (2013) menciona que involucrar conocimientos de diversas áreas del saber permite realizar un análisis completo y contextualizado del caso estudiado, lo que concuerda con los resultados de esta investigación y la enseñanza de las ciencias naturales desde el estudio de casos sobre la avifauna. Por otro lado, se coincide con Passos et al. (2007) y Niño (2012) en que el MEC lleva a relacionar diferentes puntos de vista y permite desarrollar procesos holísticos para entender los fenómenos de la naturaleza. Estas situaciones son posibles con mayor frecuencia en los contextos de básica primaria de las escuelas rurales multigrado. Sumado a lo anterior, el estudio y la observación de la avifauna promueve el gusto y el cuidado por la naturaleza, además de la integración de áreas y conocimientos para lograr un aprendizaje significativo (Mello \& Muller, 2019; Quintana, 2017; Rodríguez, 2017). 
¿Cómo la discusión de los casos promueve el diálogo y la formulación de preguntas?

En los diálogos, las preguntas son fundamentales. Según Califano y Echazú (2013) y Melo (2017), estas expresan curiosidad, conocimientos — previos, empíricos y tradicionales-, relaciones, y algunas intentan justificar algo. En esta investigación, a lo largo de las diferentes fases del método, se evidenció, en un primer instante, la prevalencia del término “ipor qué?" en las preguntas realizadas por los estudiantes. Es decir, en el estudio del primer caso - Aprendiendo con mi padre-, el docente participó en más oportunidades al formular preguntas sobre la historia para motivar a los participantes en la discusión, mientras que, en los siguientes dos casos, el papel del docente fue más de mediador de los cuestionamientos planteados por los estudiantes. Además, la secuencia de las fases del método y el ambiente generado en el aula por el docente facilitaron el reconocimiento y el uso de diferentes pronombres interrogativos: cómo, de qué manera, qué, dónde, cuántos y por qué. Sus cuestionamientos llevaron a fortalecer el análisis de los casos, al querer saber más sobre las situaciones que trataban. En palabras de Morales y Pulido (2018): "las preguntas detonan en los estudiantes la inquietud por saber más y se convierten en un mecanismo de comunicación que de una manera bidireccional involucra a maestros y estudiantes" (p. 117).

De tal manera, las preguntas realizadas por los participantes fueron mejorando y convirtiéndose en preguntas de calidad. El diálogo les permitió construir conocimientos al tiempo que desarrollaban habilidades de pensamiento. En este sentido, Calderón (2002, citado por León et al., 2018) menciona que las buenas preguntas adquieren características de lógica, precisión, claridad, capacidad para evocar más preguntas y generar curiosidad en el oyente. Las preguntas formuladas por los participantes pueden agruparse en niveles de pensamiento (Granados, 2017). Así, en la tabla 3, realizada por Arango (2020, p. 81), se muestra una clasificación de preguntas en dos de los cuatro niveles establecidos.

\section{Tabla 3}

Algunas preguntas formuladas por los estudiantes durante el estudio de cada uno de los casos agrupadas en los niveles de pensamiento

\begin{tabular}{|c|c|c|}
\hline Niveles & Acciones & Preguntas realizadas por estudiantes \\
\hline \multirow{8}{*}{$\begin{array}{l}\text { Nivel 1. Comprensión y } \\
\text { retención de información } \\
\text { sobre aves: } \\
\text { Reconocer, memorizar, } \\
\text { recordar y activar } \\
\text { información relevante. } \\
\text { Comprender la } \\
\text { información de una fuente } \\
\text { y saberla comunicar }\end{array}$} & Identificar & ¿Qué es un nombre científico? ¿Qué come el copetón? \\
\hline & Localizar & $\begin{array}{l}\text { ¿Dónde está la alondra? ¿Cuál es el norte y el sur de la } \\
\text { escuela? }\end{array}$ \\
\hline & Describir & $\begin{array}{l}\text { ¿Cómo son las patas del águila? ¿Qué tienen que tener los } \\
\text { ojos del águila para poder ver desde tan lejos? }\end{array}$ \\
\hline & Nombrar & $\begin{array}{l}\text { ¿Cómo se llama el país de donde vienen las águilas } \\
\text { pescadoras? }\end{array}$ \\
\hline & Definir & ¿Qué es endémico? \\
\hline & Explicar & $\begin{array}{l}\text { ¿Por qué a la alondra no le gustaba hacerse al lado de los } \\
\text { humanos? ¿Cómo hace el águila para comerse el pescado si } \\
\text { no tiene dientes? ¿Cómo el águila podía ver desde tan lejos } \\
\text { los peces que estaban en el fondo del lago? }\end{array}$ \\
\hline & Resumir & $\begin{array}{l}\text { ¿Por qué las aves tienen nombres científicos y de dónde } \\
\text { los sacaron? }\end{array}$ \\
\hline & Distinguir & $\begin{array}{l}\text { ¿Por qué en distintos países llamaban al copetón de } \\
\text { diferentes formas? ¿Qué otras aves realizan migración? }\end{array}$ \\
\hline
\end{tabular}




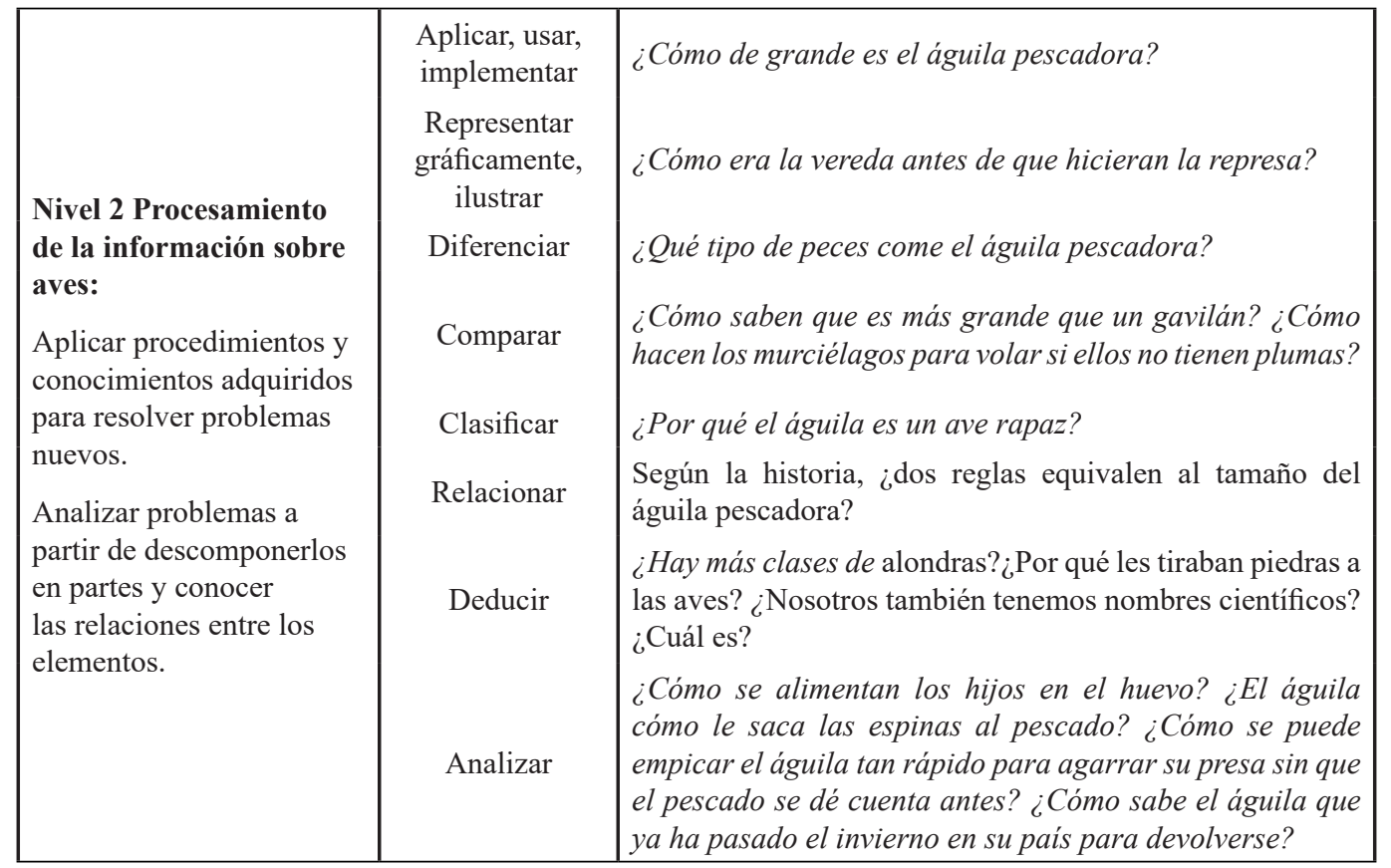

Adaptada de "La formulación de buenas preguntas en didáctica de la geografía" de J. Granados, 2017, Documents d'Anàlisi Geogràfica, 63(3).

El alcanzar estos niveles de formulación de preguntas implica que el docente también las formule y promueva su realización mediante los diálogos, con el fin de fortalecer habilidades y desarrollar tanto el pensamiento como los procesos cognitivos de los participantes. Además, las actividades diseñadas dentro del estudio de los casos traían implícitos este tipo de cuestionamientos. Así, los resultados concuerdan con Niño (2012), cuando menciona que, en el MEC, las preguntas no solo pueden ser formuladas por el docente, sino que también son construidas por los estudiantes, lo cual los invita a la reflexión y a la necesidad de saber sobre el tema.

En este sentido, el formular preguntas crea conciencia y ayuda a alcanzar una mejor comprensión de los fenómenos relacionados con las aves del contexto. Sumado a esto, a través de las preguntas, los estudiantes tuvieron una mayor participación en las sesiones de clase. A su vez, se fomentó la motivación y el diálogo. Se abrió la posibilidad de debatir sobre diferentes opiniones y perspectivas para desarrollar la toma de decisiones. Esto coincide con Granados (2017) en que las preguntas permiten comprobar supuestos, encontrar contradicciones y crear nuevo conocimiento.

Por otro lado, las fases de discusión de los casos sobre las aves de la vereda — es decir, sobre el entorno- llevaron a que las sesiones de clase se transformaran en ambientes para analizar realidades, dar ejemplos y promover actitudes de respeto y escucha (Lipman, 1991, citado por Rodríguez, 2012). También es importante señalar que el hecho de aprender del otro y con el otro lleva a que la información se comprenda y se reformulen preguntas con base en sus respuestas. De acuerdo con Niño (2012), al guiar a los participantes de esta manera, se promueve el cambio conceptual de ideas y la reacomodación de los conocimientos.

De acuerdo con los resultados, el fortalecimiento de habilidades en los estudiantes se debe a la incorporación de diversas actividades en el MEC. Esto les permite aprender con gusto, creatividad y tranquilidad, según sus ritmos. Les da la posibilidad de trabajar en 
grupos o individualmente, escribiendo o dibujando, escuchando o hablando, en un espacio donde nunca hay la presión de trabajar por una nota. Lo anterior concuerda con Cabrera y Fariñas (2005) y con Román y Ruiz (2018), quienes relacionan las diversas maneras de aprender de los estudiantes con estilos de aprendizaje, los cuales son clasificados según: las formas preferidas de los educandos para percibir y procesar la información, los modos de orientarse hacia la comunicación, la planeación de su tiempo en el cumplimiento de sus metas y sus relaciones interpersonales en el aprendizaje.

Teniendo en cuenta lo anterior, se evidenció que el MEC permite en los estudiantes el fortalecimiento de habilidades que los llevan a pensar críticamente. Esto coincide con los estudios realizados por Passos et al. (2007), Revel (2013), Gutiérrez et al. (2019) y Niño y Pedraza (2019). Además, es importante mencionar que el pensamiento crítico no se enseña individualmente y no es cuestión de una sola área (Borges et al., 2019; Rodríguez, 2012), sino de las relaciones de diversas disciplinas para comprender, analizar e interpretar los fenómenos que se presentan y se experimentan en la cotidianidad. Finalmente, el trabajo colaborativo y participativo es otra de las virtudes que es posible fomentar desde el MEC, pues se evidencia que ni la edad ni el nivel educativo son importantes a la hora de ayudar a un compañero a comprender el tema en cuestión (Vilches \& Gil, 2012). Así mismo, y como lo menciona Somma (2012), en el aula multigrado es posible adaptar el MEC a las distintas edades y niveles educativos.

\section{Conclusiones}

El MEC es una posibilidad para articular diferentes aspectos en la formación de ciudadanos. Los casos diseñados desde el contexto de los participantes - como el estudio de la avifauna- permiten analizarlo críticamente al involucrar y desarrollar diversidad de habilidades, de tal manera que se vinculan conocimientos de diferentes áreas del saber. Esto contribuye a que la enseñanza en contextos rurales con modalidad multigrado promueva un aprendizaje significativo, creativo, valioso, colaborativo y útil en sus estudiantes.

Reconocer parte de las especies de aves que habitan en la vereda Leonera de Toca permitió diseñar los diferentes casos de estudio y utilizar la enseñanza de las ciencias para fomentar el desarrollo de habilidades como la expresión escrita, formular preguntas, observar, discutir, argumentar, construir conceptos, reflexionar, comunicar, entre otras, así como el desarrollo de valores para convivir en sociedad, como el saber escuchar y hablar, ya que son elementos básicos para establecer un diálogo, el estar atentos a los diferentes aportes, el reconocer las opiniones, el saber evaluar los argumentos propios y de los otros y el respetar la palabra del otro ${ }^{1}$.

\section{Referencias}

Abós, P. (2015). El modelo de escuela rural multigrado ¿es un modelo del que podamos aprender? ¿es transferible a otro tipo de escuela? Innovación Educativa, (24), 99-118. https://docplayer.es/42937485-El-modelo-de-escuela-rural-multigrado-es-un-modelodel-que-podamos-aprender-es-transferible-a-otro-tipo-de-escuela.html

1 Este artículo es producto del proyecto de investigación Una Mirada al Currículo desde la enseñanza aprendizaje de las Ciencias Naturales en Básica Primaria. Desarrollado por el grupo de investigación GECOS. SGI 2693 DIN UPTC. 
Abós, P., Torres, C., \& Fuguet, J. (2017). Aprendizaje y escuela rural: la visión del alumnado. Sinéctica, Revista Electrónica de Educación, (46), 1-17. http://www.scielo.org.mx/pdf/ sine/n49/2007-7033-sine-49-00006.pdf

Arango, A. (2020). Aprendiendo sobre aves: una estrategia para el desarrollo de habilidades de pensamiento crítico y la enseñanza en escuela rural multigrado [Tesis de pregrado, Universidad Pedagógica y Tecnológica de Colombia]. Repositorio UPTC. https:// repositorio.uptc.edu.co/handle/001/3153

Argandoña, F., Persico, M., \& Visic, A. (2018). Estudio de caso: una metodología de enseñanza en la educación superior para la adquisición de competencias integradoras y emprendedoras. TEC Empresarial, 12(3), 7-16. https://www.scielo.sa.cr/pdf/tec/ v12n3/1659-3359-tec-12-03-7.pdf

Arias, J. (2017). Problemas y retos de la educación rural colombiana. Revista Educación y Ciudad, (33), 53-62. https://doi.org/10.36737/01230425.v0.n33.2017.1647

Arriagada, C., \& Calzadilla, O. (2018). Percepción de las bases curriculares de la educación básica multigrado en la Araucanía, Chile. Praxis \& Saber, 9(20), 75-95. https://doi. org/10.19053/22160159.v9.n20.2018.8296

Barros, A. (2019). Aplicación del método de casos en la educación para la sexualidad. Biografía, (Número extraordinario: Memorias del X Encuentro Nacional de Experiencias en Enseñanza de la Biología y la Educación Ambiental), 618-623. https://revistas. pedagogica.edu.co/index.php/bio-grafia/article/view/10959

Barros, A., Méndez, C., \& Saavedra, J. (2017). La enseñanza del sistema nervioso mediante la estrategia didáctica del método de caso para la prevención de las adicciones al alcohol y el tabaco [Tesis de maestría, Fundación Universidad del Norte]. http://manglar.uninorte. edu.co/bitstream/handle/10584/7955/131416.pdf? sequence=1\&isAllowed=y

Borges, P., Souza de, M., Aparecida, P., Aline, G., \& Olivo, A. (2019). O pensamento crítico nos planos de aula direcionados para a educação ambiental. Bio-grafía, (Número extraordinario: Memorias del X Encuentro Nacional de Experiencias en Enseñanza de la Biología y la Educación Ambiental), 1202-1213. https://revistas.pedagogica.edu.co/ index.php/bio-grafia/article/view/11068

Bustos, A. (2014). La didáctica multigrado y las aulas rurales: perspectivas y datos para su análisis. Innovación Educativa, (24), 119-131. https://dialnet.unirioja.es/servlet/ articulo? codigo $=4920455$

Cabrera, J., \& Fariñas, G. (2005). El estudio de los estilos de aprendizaje desde una perspectiva vigostkiana: una aproximación conceptual. Revista Iberoamericana de Educación, 37(1), 1-10. https://doi.org/10.35362/rie3712731

Califano, L., \& Echazú, F. (2013). Etnobotánica en comunidades pastoriles. Conocimiento tradicional sobre especies tóxicas para el ganado en la cuenca del río Iruya (Salta, Argentina). Bol. Soc. Argent. Bot., 48(2), 365-375. https://botanicaargentina.org.ar/wpcontent/uploads/2017/05/18_califano.pdf

Chin, V. (2013). Aplicación de la estrategia didáctica "estudio de casos" para un curso de biología general en la Universidad Nacional de Colombia sede Medellín [Tesis de Maestría, 
Universidad Nacional de Colombia]. Repositorio institucional UN. http://bdigital.unal. edu.co/9620/1/43751586.2013.pdf

Colbert, V. (1999). Mejorando el acceso y la calidad de la educación para el sector rural pobre: El caso de la Escuela Nueva en Colombia. Revista Iberoamericana de Educación, (20), 107-135.

Díaz, F., \& Hernández, G. (1999). Estrategias de enseñanza para la promoción de aprendizajes significativos ( $3^{\mathrm{a}} \mathrm{ed}$.). McGraw-Hill.

Díaz, S., Mendoza, V., \& Porras, C. (2011). Una guía para la elaboración de estudio de casos. Razón y Palabra, (75). http://www.razonypalabra.org.mx/N/N75/varia_75/01_Diaz_ V75.pdf

Faure, I. (2017). Componentes del proceso de enseñanza aprendizaje en las escuelas multigrados. EduSol, 17(61).https://dialnet.unirioja.es/servlet/articulo?codigo=6137070

Gamboa, M. (2017). Estudio de caso como estrategia didáctica para el proceso enseñanzaaprendizaje: retos y oportunidades. Bio-grafía, 10(19), 1533-1540. https://doi. org/10.17227/bio-grafia.extra2017-7334

Gil, J., \& Barreto, C. (2019). El caso situacional como estrategia de enseñanza para el desarrollo de la competencia argumentación científica en educación universitaria. Biografía, (Número extraordinario: Memorias del X Encuentro Nacional de Experiencias en Enseñanza de la Biología y la Educación Ambiental), 441-453. https://revistas. pedagogica.edu.co/index.php/bio-grafia/article/view/10877

Granados, J. (2017). La formulación de buenas preguntas en didáctica de la geografía. Documents d’Anàlisi Geogràfica, 63(3), 545-559. http://dx.doi.org/10.5565/rev/dag.495

Gutiérrez, H., Chamizo, J., Cano, E., \& Armany, N. (2019). El método del caso en la formación de economistas: elaboración y aplicación. Magis, 12(23), 145-168. http:// dx.doi.org/10.11144/Javeriana.m12-25.emdc

Hernández, R., Fernández, C., \& Baptista, P. (2014). Metodología de la investigación (6ª ed.). Mc Graw Hill.

Herrera, L., \& Buitrago, R. (2015). Educación rural en Boyacá, fortalezas y debilidades desde la perspectiva del profesorado. Praxis \& Saber, 6(12), 169-190. https://doi. org/10.19053/22160159.3768

Jiménez, V., \& Comet, C. (2016). Los estudios de casos como enfoque metodológico. ACADEMO Revista de Investigación en Ciencias Sociales y Humanidades, 3(2). https:// dialnet.unirioja.es/servlet/articulo? codigo $=5757749$

Lacruz, J., Cebrián, V., \& Fernández, M. (2017). Expectativas y creencias del alumnado rural sobre su futuro profesional y académico. Aula Abierta, (45), 49-54. http://dx.doi. org/10.17811/rifie.45.2017.49-54

Lara, P., \& Pulido, Ó. (2020). Escritura como práctica de sí y escuela rural. Praxis \& Saber, 11(25), 21-45. https://doi.org/10.19053/22160159.v11.n25.2020.10480

León, F., Duque, E., \& Escobar, P. (2018). Estrategias de formulación de preguntas de calidad 
mediadas por realidad aumentada para el fortalecimiento del pensamiento científico. Revista Mexicana de Investigación Educativa, RMIE, 23(78), 791-815. http://www.scielo. org.mx/pdf/rmie/v23n78/1405-6666-rmie-23-78-791.pdf

Martínez, L. (2007). La observación y el diario de campo en la definición de un tema de investigación. Perfiles Libertadores, 4(80), 73-80.

Mello, D., \& Muller, E. (2019). Influência das unidades de conservação sobre a percepção dos estudantes da educação básica em relação às aves. Rev. Eletrônica Mestr. Educ. Ambient, 36(1), 305-323.

Melo, N. (2017). Los puentes en la enseñanza de las ciencias: un compromiso para comprender las investigaciones sobre las relaciones entre conocimientos científicos escolares y conocimientos ecológicos tradicionales. Tecné, Episteme y Didaxis: TED, (42), 43-61. http://www.scielo.org.co/pdf/ted/n42/0121-3814-ted-42-00043.pdf

MEN. (2002). Diagnóstico del Plan Nacional de Desarrollo 2002-2006 "Hacia un Estado Comunitario": Portafolio de Modelos Educativos. Dirección de Poblaciones y Proyectos Intersectoriales. https://www.mineducacion.gov.co/1621/article-89618.html

MEN. (2010). Escuela Nueva. Orientaciones Pedagógicas de Segundo a Quinto Grado, Tomo II. http://aprende.colombiaaprende.edu.co/per/87095

Morales, L., \& Pulido, Ó. (2018). Ambientes filosóficos para la lectura en la escuela rural. Praxis \& Saber, 9(21), 99-124. https://doi.org/10.19053/22160159.v9.n21.2018.8925

Niño, L. (2012). Estudio de caso: una estrategia para la enseñanza de la educación ambiental. Praxis \& Saber, 3(5), 53-78. https://doi.org/10.19053/22160159.1133

Niño, L., \& Pedraza, Y. (2019). Potencias de la educación ambiental a través del estudio de caso. TED, (45), 143-158. http://www.scielo.org.co/pdf/ted/n45/0121-3814ted-45-00143.pdf

Passos, L., Andretta, C., \& Linhares, S. (2007). Estudos de caso em química. Quim. Nova, 30(3), 731-739. http://static.sites.sbq.org.br/quimicanova.sbq.org.br/pdf/Vol30No3_731_38ED06200.pdf

Piñones, C., \& Zuleta, C. (2015). Conociendo las aves y los humedales del desierto costero de Chile: experiencias con una academia escolar en los Vilos. Bio-grafía, 8(15), 137-147. https://doi.org/10.17227/20271034.vol.8num.15bio-grafia136.147

Quintana, R. (2017). La educación ambiental y su importancia en la relación sustentable: Hombre-Naturaleza-Territorio. Revista Latinoamericana de Ciencias Sociales, Niñez y Juventud, 15(2), 927-949. https://doi.org/10.11600/1692715x.1520929042016

Ramírez, F., Blanquicet, R., \& Ramírez, N. (2019). Docentes no licenciados en ciencias naturales: un acercamiento a sus concepciones, en el marco de la educación rural. Biografía, (Número extraordinario: Memorias del X Encuentro Nacional de Experiencias en Enseñanza de la Biología y la Educación Ambiental), 567-577. https://revistas. pedagogica.edu.co/index.php/bio-grafia/article/view/10954

Revel, A. (2013). Estudios de caso en la enseñanza de la biología y en la educación para la salud en la escuela media. Bio-grafía, 6(10), 42-49. https://doi.org/10.17227/20271034. 
vol.6num.10bio-grafia42.49

Rivera, Á., \& Zabala, M. (2019). Construcción de alternativas a problemáticas sentidas. Revisión de modelo educativo para el medio rural. RLEEI, 3(4), 70-86.

Rodríguez, D. (2017). Sobrevolando el mundo de las aves: una estrategia en la enseñanza y la conservación de las aves. Bio-grafía, 10(18), 63-73. https://doi.org/10.17227/20271034. vol.10num.18bio-grafia63.73

Rodríguez, M. (2012). Pensamiento crítico y aprendizaje: una competencia de alto nivel en la Educación Básica. Limusa.

Román, E., \& Ruíz, V. (2018). Estilos de aprendizaje y su relación con el rendimiento académico de estudiantes de segundo año de educación general básica. Revista de Estilos de Aprendizaje, 11(22), 166-178. http://revistaestilosdeaprendizaje.com/article/ view/1084

Sanabria, A., \& Sosa, P. (2017). Habilidades científicas a través del conocimiento de las aves colombianas. Enseñanza de las Ciencias, (Número extraordinario: X Congreso Internacional sobre Investigación en Didáctica de las Ciencias), 1071-1075. https://ddd. uab.cat/pub/edlc/edlc_a2017nEXTRA/39_-_Habilidades_Cientificas_a_traves_del_ conocimiento_de_las_aves_Colombianas.pdf

Somma, L. (2012). El estudio de casos. Una estrategia de construcción de aprendizaje. Reflexión Académica en Diseño y Comunicación, 14(21), 32-34. https://fido.palermo.edu/ servicios_dyc/publicacionesdc/archivos/430_libro.pdf

Soto, D., \& Molina,L. (2018). La escuela rural en Colombia como escenario deimplementación de TIC. Saber, Ciencia y Libertad, 13(1), 275-289. https://doi.org/10.18041/2382-3240/ saber.2018v13n1.2086

Strauss, A., \& Corbin, J. (2002). Bases de la investigación cualitativa. Técnicas y procedimientos para desarrollar la Teoría Fundamentada. Universidad de Antioquia. https://diversidadlocal.files.wordpress.com/2012/09/bases-investigacion-cualitativa.pdf

Vilches, A., \& Gil, D. (2012). El trabajo cooperativo en el aula. Una estrategia considerada imprescindible pero infrautilizada. Aula de Innovación Educativa, (208), 41-46. http:// crateru1.educa.aragon.es/trabajo_cooperativo_revista_aula208.pdf

Wassermann, S. (1994). El estudio de casos como método de enseñanza. Amorrortu. http:// terras.edu.ar/biblioteca/3/3EEDU_Waserman_1_Unidad_2.pdf

Wassermann, S. (2006). El estudio de casos como método de enseñanza. Amorrortu. 